\title{
Usefulness of basic laboratory parameters for monitoring during the perianesthetic period for selected ophthalmic operations in mesaticephalic and brachycephalic dogs
}

\author{
AGNIESZKA WRZESIŃSKA*, WŁODZIMIERZ KLUCIŃSKI ${ }^{* *}$, MAGDALENA GARNCARZ** \\ **Division of Laboratory and Clinical Diagnostics, Department of Pathology and Veterinary Diagnostics, \\ Warsaw University of Life Sciences - SGGW, ul. Nowoursynowska 159 C, 02-776, Warsaw \\ *Ophthalmology Veterinary Clinic, ul. Grupy Armii Krajowej Północ 2/10, 00-713 Warsaw
}

Wrzesińska A., Kluciński W., Garncarz M.

Usefulness of basic laboratory parameters for monitoring during the perianesthetic period for selected ophthalmic operations in mesaticephalic and brachycephalic dogs

\section{Summary}

The aim of this study was to compare the usefulness of laboratory diagnostic parameters dedicated for general anesthesia in brachycephalic and mesaticephalic dogs. The study was performed on two groups of dogs of 112 animals each, representing two different morphotypes, brachycephalic and mesaticephalic ones, which underwent general anesthesia with medetominidine, midazolam, propofol and isoflurane. Before and after anesthesia, the dogs underwent basic haematological and biochemical tests. In $18 \%$ of mesaticephalic dogs and $16 \%$ of brachycephalic dogs, the results of laboratory blood tests show a significant increase in the activity of liver enzymes: aspartate aminotransferase (AST), alanine phosphatase (ALT) and alkaline phosphatase (ALP), even though the animals showed no clinical signs other than those recognized in ophthalmologic examination. This demonstrates the presence of subclinical hepatic changes in these animals. Moreover, general anesthesia had a greater negative impact on liver function if liver enzyme values had exceeded certain levels before the anesthesia (AST >61 U/L, ALT >91 U/L). This made it possible to define new, useful upper limit values of AST and ALT for subclinical dogs before anesthesia that could lead to more serious complications of liver function after anesthesia. Among all the examined parameters, it is beneficial to monitor the activity of AST, ALT and ALP during the perianesthetic period. In the postanesthesia monitoring, creatinine concentration should also be included.

Keywords: brachycephalic morphotype, mesaticephalic morphotype, general anesthesia, dog

General anesthesia, as a controllable and reversible process of consciousness abolition and analgesia, causes a temporary destabilization of physiological functions. The temporary inhibition of central nervous system functions, with preservation of respiratory and circulatory functions, is associated with a risk of complications, including death. The overriding element of every anesthetic protocol in companion animals is minimizing the risk of complications. Two procedural models have been developed for this purpose. The first calls for performing blood tests when clinical examinations reveal abnormalities or when additional risk factors are recognized (e.g. age). The second model calls for similar blood tests irrespective of the patient's clinical state or presence of additional risk factors in order to diagnose disease at a subclinical stage (1). Additional risk factors in anesthesia of the canine patient include age (geriatric or neonatal), weight (obesity), and morphotype (specifically brachycephalic dogs). Mesaticephalic dog breeds are considered the least hindered in terms of their skull morphology by specific breeding programs. This group includes highly selected breeds such as the Siberian Husky (the breed least modified by man) and the English Cocker Spaniel. Mesaticephalic dog breeds may have characteristic diseases, for example, entropiom in Polish Hounds or English Cocker Spaniels. In addition, the polymorphism of dogs can predispose to anatomical 
abnormalities (e.g. abnormal eyelid position requiring surgical correction under general anesthesia $(8,16$, 17,21 ) or clinical abnormalities (e.g. impaired gas exchange or dyspnoea during or after anesthesia (2)), such as are seen in the brachycephalic dog breeds. These latter abnormalities are related to the local chondrodysplasia of the facial aspect of the skull created by breeding selection (14). The cartilage of the skull base undergoes ankylosis during the early period of development. This predisposes to the shortening of the long axis of the skull bones while at the same time allowing them to grow in width (12). The facial and occipital skull bones become deformed, causing a myriad of undesirable medical traits collectively referred to as BAOS (brachycephalic obstructive airway syndrome) (22). This syndrome includes soft palate elongation, stenotic nares, an increased amount of tissue in the throat and submandibular area, laryngeal collapse, tracheal hypoplasia, everted laryngeal saccules with mucous membrane edema and hyperplasia $(6,20$, $25)$. Because brachycephalic dogs are predisposed to $\mathrm{BAOS}$, it is assumed that the risk of general anesthesia for the whole group is increased $(3,13)$. In fact, BAOS occurs frequently, but not in $100 \%$ of brachycephalic dogs (18). According to Nijakama et al (18), it is seen most frequently in Bulldogs and Pugs. In a population of $2207 \mathrm{dogs}$, BAOS was observed in $44 \%$ of Bulldogs but in no Boxers. Although the brachycephalic morphotype includes over a dozen dog breeds, only 3 are predisposed to BAOS: Pugs, English Bulldogs and French Bulldogs (5). It is therefore debatable whether all dogs belonging to the brachycephalic morphotype should be considered at increased risk for general anesthesia.

Changes occurring during general anesthesia may persist after its completion. These include hypotension, a decrease in cardiac output and stroke volume and the resulting impaired organ perfusion, as well as possible transient tissue hypoxia $(10,15,23)$. Post-anesthesia monitoring in the form of control tests is therefore

Tab. 1. The numbers of dogs of particular breeds in the mesaticephalic and brachycephalic groups

\begin{tabular}{|l|c|l|c|}
\hline \multicolumn{1}{|c|}{ Mesaticephalic breeds } & $\begin{array}{c}\text { Number } \\
\text { of dogs }\end{array}$ & Brachycephalic breeds & $\begin{array}{c}\text { Number } \\
\text { of dogs }\end{array}$ \\
\hline West Highland White Terrier & 11 & Pekingese & 9 \\
\hline Miniature Schnauzer & 12 & Shih Tzu & 11 \\
\hline Pembroke Welsh Corgi & 7 & Cavalier King Charles Spaniel & 4 \\
English Cocker Spaniel & 11 & French Bulldog & 8 \\
\hline Polish Hunting Dog & 27 & English Bulldog & 13 \\
Newfoundland Dog & 10 & Shar-pei & 23 \\
Polish Hound & 4 & Chow-chow & 14 \\
Leonberger & 4 & Boxer & 9 \\
St. Bernard & 7 & American Bulldog & 7 \\
Mesaticephalic morphotype & 19 & Bullmastiff & 10 \\
crossbreed & & Dogue de Bordeaux & 4 \\
\hline
\end{tabular}

recommended, but no precise time frame is given. In addition, if test results show elevated values prior to anesthesia, it is unclear how to proceed: whether the procedure under general anesthesia should be performed or postponed, if the choice is available. It is interesting to note whether there are laboratory blood parameter anomalies related to changes caused by general anesthesia in the brachycephalic morphotype group, especially since it is made up of a large number of dog breeds without BAOS. The aim of this study was to evaluate the diagnostic usefulness of selected laboratory parameters in dogs of mesaticephalic and brachycephalic morphotypes during the perianesthetic period for specific ocular procedures.

\section{Material and methods}

Animals. The study was performed on a population of 224 privately owned dogs (112 mesaticephalic and 112 brachycephalic) that underwent ophthalmic surgery under general anesthesia. The owner of each dog gave written consent for the surgery and for including the animal in the study. Dogs of both sexes, different breeds, ages 8 months to 5 years, weighing $5-80 \mathrm{~kg}$ were included. A 5-point body condition score (BCS) was used, and only dogs with a $3 / 5$ score were included (neither skinny nor obese). Inclusion criteria included the absence of clinical symptoms seen directly in the clinical examination and the absence of symptoms of BAOS in the brachycephalic dogs. All dogs were subjected to general anesthesia according to the same scheme lasting $70.00 \pm 5.00 \mathrm{~min}$. The ophthalmic surgeries, with similar trauma, included correction of eyelid and cilia abnormalities and lasted $60.00 \pm 8.00 \mathrm{~min}$. The numbers of dogs of particular breeds belonging to both morphotypes are shown in Table 1.

General anesthesia procedure. The dogs were not fed for 12 hours and not given water for 3 hours prior to general anesthesia. General anesthesia was performed in 3 stages: premedication with medetomidine hydrochloride (Cepetor, $1 \mathrm{mg} / \mathrm{ml}$, Scanvet) given at a dose of $0.01 \mathrm{mg} / \mathrm{kg}$ in combination with midazolam (Midanium, $5 \mathrm{mg} / \mathrm{ml}$, Polfa) at a dose of $0.2 \mathrm{mg} / \mathrm{kg}$ given in 1 intramuscular injection; induction for intubation with propofol (Plofed $1 \%, 10 \mathrm{mg} / \mathrm{ml}$, Polfa) at an average dose of $1 \mathrm{mg} / \mathrm{kg}$; and maintenance by semi-opened inhalation (Isofluranum 100\%, Aerrane, Baxter) diluted in $100 \%$ medical oxygen at a volume of $1.5 \%$ using an anesthetic machine for small animals (SurgiVet CDS 9000). Analgesia was obtained with topical proxymetacaine hydrochloride (Alcaine, Proxzmetacainihzdrochloridum $0.5 \%$, Alcom) for local analgesia and fentanyl (Fentanylum $50 \mu \mathrm{g} / \mathrm{ml}$, Fentanyl WZF, Polfa) diluted in $0.9 \% \mathrm{NaCL}$ solution (Natriumchloratum 0,9\%, $100 \mathrm{ml}$, Baxter) given at a continuous rate $(2 \mu \mathrm{g} / \mathrm{kg} / \mathrm{h})$ for central analgesia. The animals woke up from anesthesia without the aid of antagonistic agents, being allowed to spontaneously breathe atmospheric air after disconnection of the anesthetic machine. They were placed in sternal recumbency on a heating pad with their heads slightly elevated. The tracheal tube was removed immediately 
after the animal regained consciousness and regained the swallowing and cough reflexes.

Blood collection for laboratory testing. Blood samples from all animals were collected from the cephalic vein 24 hours before and 24 hours after general anesthesia. In accordance with generally accepted principles, blood was drawn into two tubes: one without an anticoagulant to obtain serum for biochemical tests and the other with EDTA-K2 anticoagulant for hematological analysis.

Determination of laboratory parameters. Red and white blood cell parameters as well as platelets were determined with an Abacus Junior Vet hematologic analyzer. The following parameters were included in the analysis: the number of erythrocytes (red blood cells, RBC), hematocrit $(\mathrm{Ht})$, hemoglobin $(\mathrm{Hb})$, mean corpuscular volume (MCV),

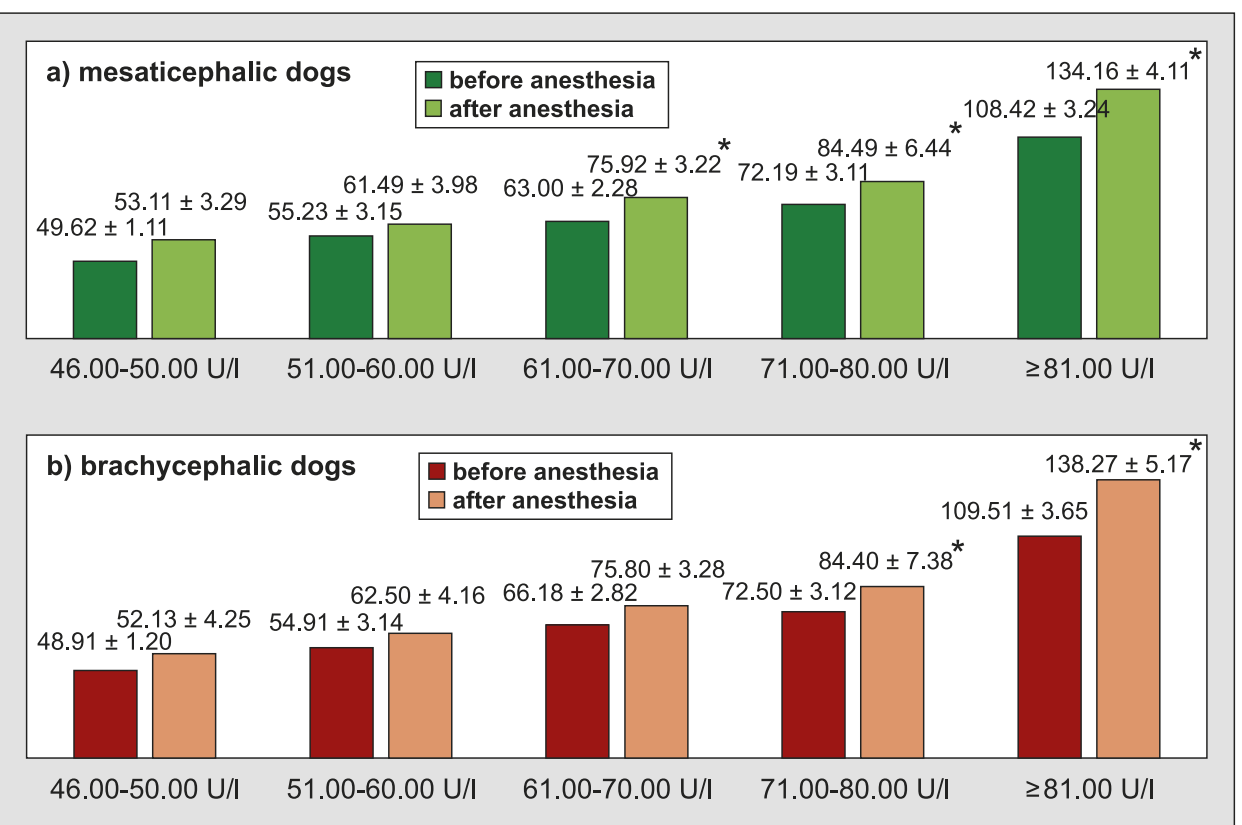

Fig. 1. Mean serum activities of aspartate aminotransferase 24 hours before and after general anesthesia in the mesaticephalic group (a) and in the brachycephalic group (b)

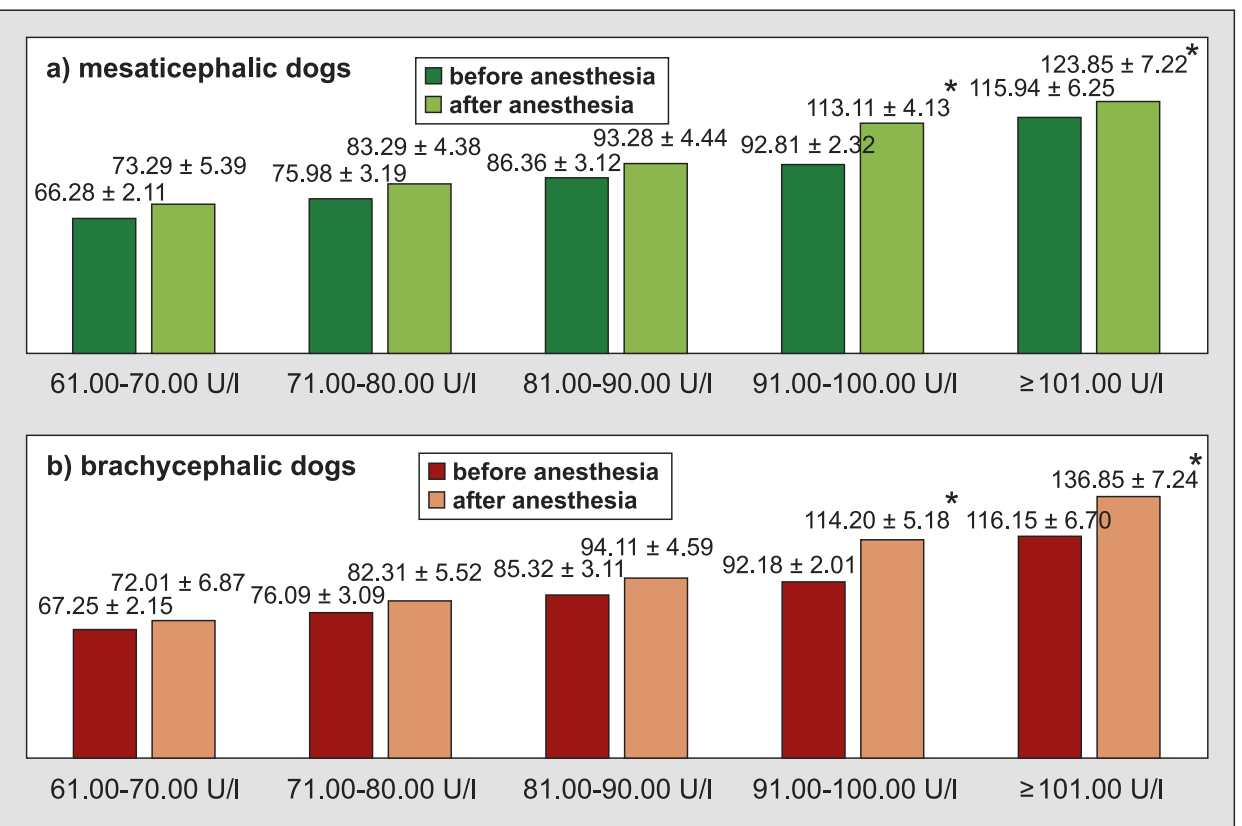

Fig. 2. Mean serum activities of alanine aminotransferase 24 hours before and after general anesthesia in the mesaticephalic group (a) and in the brachycephalic group (b) mean corpuscular hemoglobin $(\mathrm{MCH})$, mean corpuscular hemoglobin concentration (MCHC), platelet count (PLT), and leukocyte count (WBC). The leucogram included the number and percentage of lymphocytes (LYM, LY\%), the number and percentage of monocytes (MON, MON\%), and the number and percentage of granulocytes(GRA, GR\%). Biochemical studies were performed with a Pointe 180 plus device to analyze the activity of the following parameters in the serum: alanine aminotransferase (ALT), aspartate aminotransferase (AST), alkaline phosphatase (ALP) and the concentrations of glucose, urea, creatinine and total protein.

For complete visualization of enzyme activity before and after general anesthesia, statistical analysis was performed after dividing each morphotype group into 5 subgroups according to their AST values in 10-unit increments. ALP values were divided into 2 subgroups within each morphotype group.

The statistical analysis was performed using the SPSS 14.0 statistical package GLM. The relationship between particular parameters obtained before, during and after anesthesia was determined by the $\chi^{2}$ test.

\section{Results and discussion}

There were no statistically significant differences in hematological parameters measured 24 hours before general anesthesia for one of the four ophthalmic surgeries in dogs of both morphotypes. All values for RBC, WBC and PLT were within reference values. There were no significant differences in these values 24 hours after anesthesia completion and ophthalmic surgery compared to the initial values before anesthesia. Gender, age and weight did not have an influence on these parameters in dogs of both morphotype $(p>0.05)$. There were also no significant differences in serum glucose, total protein and urea concentrations between the two morphotypes 24 hours before and after general anesthesia. The average glucose concentration in both morphotype groups before and after anesthesia was $4.92 \pm$ $0.19 \mathrm{mmol} / 1$, total protein concentration was $67.92 \pm 0.43 \mathrm{~g} / \mathrm{l}$, and urea was $6.42 \pm 0.20 \mathrm{mmol} / \mathrm{l}$. Figures $1 \mathrm{ab}, 2 \mathrm{ab}$ i $3 \mathrm{ab}$ show the average serum activities of AST, ALT and ALP 24 hours before and after general anesthesia with subgroups prior to anesthesia. Dogs 


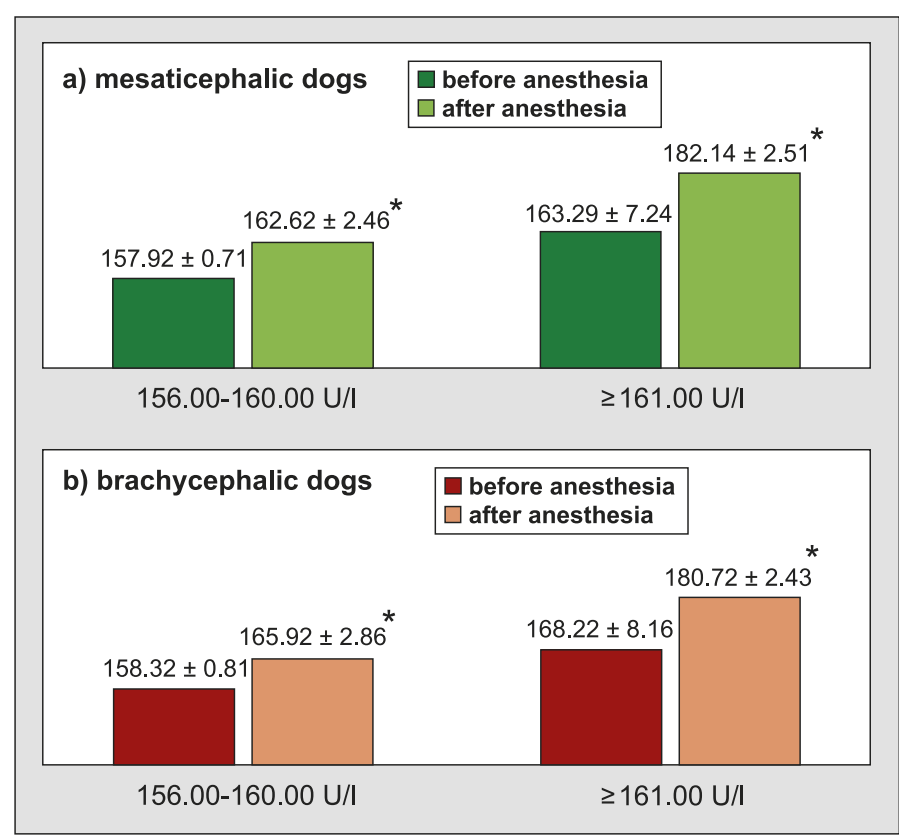

Fig. 3. Mean serum activities of alkaline phosphatase 24 hours before and after general anesthesia in the mesaticephalic group (a) and in the brachycephalic group (b)

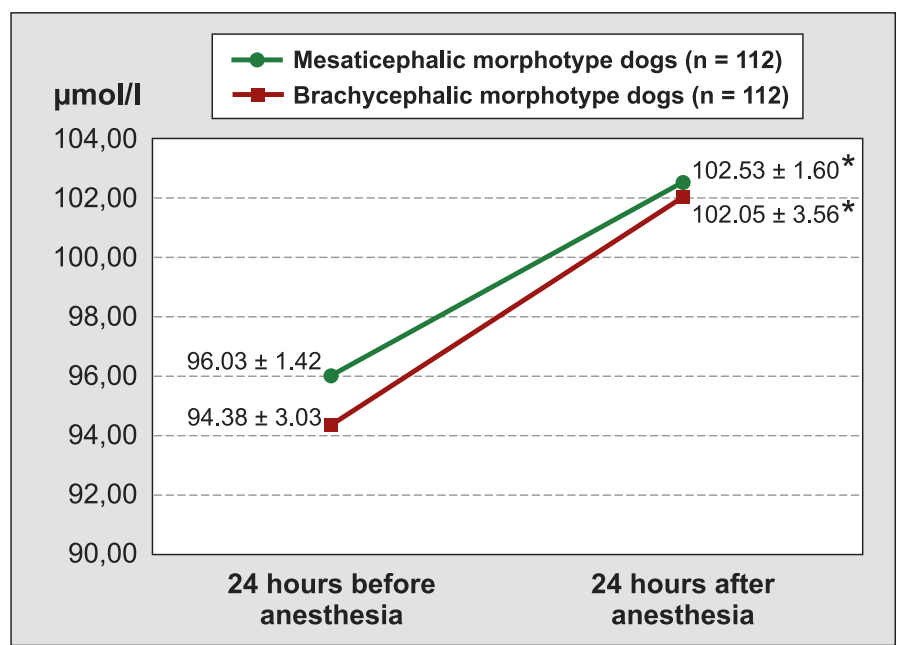

Fig. 4. Mean creatinine concentrations 24 hours before and after anesthesia in the mesaticephalic and brachycephalic groups

of both morphotypes showed a significant increase in AST, ALT and ALP activities 24 hours after general anesthesia compared to values before anesthesia if the initial values were higher than 61.0, 91.0, and 156.0 $\mathrm{U} / 1$ for AST, ALT and ALP, respectively. A significant increase in serum creatinine concentration 24 hours after anesthesia was also recorded in dogs of both morphotypes compared to initial concentrations (Fig. 4).

The results of this study partially overlap with data from the literature. According to the literature (10), increased hematocrit and hemoglobin concentrations in brachycephalic dog breeds resulting from reduced partial oxygen pressure secondary to hypoxia were documented only in dogs with advanced BAOS. The comparative analysis carried out by the authors suggests that the basic hematological values in brachycephalic dogs without airway obstruction typical of
BAOS did not differ significantly from values obtained for mesaticephalic breeds (26). In the present study, no significant differences were observed in the hematological values obtained 24 hours after anesthesia, which indicates the absence of anesthetic effect in both morphotype groups and underlines that general anesthesia can be performed without higher risk in brachycephalic dogs.

Similar results were obtained when analyzing WBC values, which were within reference ranges before and after anesthesia in both morphotype groups. The ophthalmic diseases in both the brachycephalic and mesaticephalic dogs were related to eyelid defects and cilia abnormalities. They were not responsible for generalized inflammation that could be seen in the form of leukocytosis on the leukogram. The inflammation was localized to the eye (conjunctivitis) in both groups of dogs. None of the dogs showed corneal disease or trauma, that is, corneal ulcers. These observations were consistent with the Read and Broun study (21), which shows that entropion did not cause significant inflammation resulting from surgical correction of the eyelid. Inflammation secondary to surgical tissue damage and postoperative stress is minimal during eyelid or cilia correction and does not cause a strong inflammatory response seen in the leukogram, as confirmed by the results of this study. The less traumatic the surgical intervention, the less inflammatory response is seen (11). An additional factor that may significantly influence the inflammatory response is the brachycephalic syndrome in short-nosed breeds. Dogs with advanced BAOS may have elevated concentrations of inflammatory factors, such as TNF- $\alpha$, IL-10 and IL-13. Therefore, the diagnostic value of white blood cells in brachycephalic dogs without BAOS or clinical signs other than the ophthalmic signs of disease, is limited in the pre- and post-anesthetic evaluation.

There were no significant differences between the numbers of brachycephalic and mesaticephalic dogs with elevated AST, ALT, ALP. AST and ALT were elevated in $0.01 \%$ of brachycephalic and mesaticephalic dogs, while ALP in $0.02 \%$ of the study population (26). The remaining biochemical values did not differ. Therefore the risk of subclinical changes found with the aid of biochemical tests is the same for brachycephalic and mesaticephalic breeds. Out of the biochemistry parameters tested, AST, ALT and ALP were diagnostically useful in both morphotypes. These observations confirm that anesthetic agents have an indirect effect on hepatic cells and function by decreasing blood pressure and causing hepatic ischemia and hypoxia $(4,9$, 19, 24). This study also reveals a correlation between liver enzyme activity and anesthesia: the greater the activity of liver enzymes before anesthesia, the higher the values will be after anesthesia. The significant effect of general anesthesia on AST and ALT activities when these exceed $>61 \mathrm{U} / 1$ and $>91 \mathrm{U} / 1$, respectively, should be useful in determining new practical upper 
limits of these enzymes in dogs before anesthesia. The present study underlines the importance of determining the increase in liver enzyme activity by showing that with a more significant increase in these parameters before anesthesia, a more severe increase occurs after anesthesia (26). In addition, if the enzyme activity exceeds the reference range, but is not higher than the level mentioned above, ophthalmic operations can be carried out without delay without causing significant changes. Because the present study included only a small number of dogs with ALP activity significantly exceeding the reference range, a study on a larger population of dogs should be carried out in order to prove the same type of correlation. It must be underlined that the morphotype of the breed had no influence on these results, as similar tendencies were noted in both groups. This study shows that despite the proven significant indirect effect of anesthesia on the kidneys via hypotension, as well as decreased blood flow and glomerular filtration in the kidneys (7), general anesthesia by accepted methods can be performed safely without detriment to the kidneys. These changes occur regardless of the morphotype and are comparable in severity in both groups of dogs.

This study shows that, out of the laboratory parameters analyzed in both morphotype groups, it is beneficial to monitor the activities of AST, ALT and ALP during the perianesthetic period for ophthalmic eyelid and cilia correction surgeries. The results of this study suggest that it would be beneficial to take into account new values of these enzymes, below which anesthesia can be performed without harm to the patient.

\section{References}

1. Alef M., Von Praum F., Oechtering G.: Is routine pre-anaesthetic haematological and biochemical screening justified in dogs? Vet. Anesth. and Analg. J. 2008, 35, 132-140

2. Amis T. C., Kurpershoek C.: Pattern of breathing in brachycephalic dogs Am. J. Vet. Res. 1986, 47, 2200-2204.

3. Brodbelt D. C., Hammond R., Tuminaro D., Pfeiffer D. U., Wood J. L. N.: Risk factors for anaesthetic-related death in referred dogs. Vet. Rec. 2006, $158,563-564$

4. Court M. H., Hay-Kraus B. L., Hill D. W., Kind A. J., Greenblatt D. J.: Propofol hydroxylation by dog liver microsomes: assay development and dog breed differences. Drug. Metab. Dispos. 1999, 27, 1293-1299.

5. Fasanella F. J., Shivley J. M., Wardlaw J. L.: Brachycephalic airway obstructive syndrome in dogs: 90 cases (1991-2008). J. Am. Vet. Med. Ass. 2010, 237, 1048-1051.

6. Freiche $V$., Poncet C.: Górne drogi oddechowe i syndrom żołądkowo-jelitowy u psów ras brachycefalicznych. Vet. Focus 2007, 17, 4-10.

7. Fusellier M., Desfontis J. C., Madec S., Gautier F., Debailleul M., Gogny M.: Influence of three anesthetic protocols on glomerular filtration rate in dogs. Am. J. Vet. Res. 2007, 68, 807-811.

8. Gough A., Thomas W.: Breed Predispositions to Disease in Dogs and Cats. Wiley-Blackwell, United Kingdom 2010.

9. Hammond R. A., England G. C. W.: The effect of medetomidine premedication upon propofol induction and infusion anaesthesia in the dog. Vet. Anaesth. Analg. 1994, 21, 24-28.

10. Hoareau G. L., Jourdan G., Mellema M., Verwaerde P.: Evaluation of arterial blood gases and arterial blood pressures in brachycephalic dogs. J. Vet. Intern. Med. 2012, 26, 897-904.

11. Kehlet H.: Surgical stress response: does endoscopic surgery confer an advantage? World J. Surg. 1999, 23, 801-807.

12. Koch D. A., Arnold S., Hubler M., Montavon P. M.: Brachycephalic syndrome in dogs. Small Anim. Comp. 2003, 25, 48-54.
13. Krein S., Wetmore L. A.: Breed-Specific Anesthesia. Complications NAVC Clinician's Brief. 2012, 17-20.

14. Kupczyńska M., Wąsowicz M., Barszcz K., Pobłocki P., Michalczuk A.: Przyżyciowe kryteria morfometryncze głowy psów brachycefalicznych. Med. Weter. 2008, 64, 702-706.

15.Laubie M., Schmitt H., Canellas J., Roquebert J., Demichel P.: Centrally mediated bradycardia and hypotension induced by narcotic analgesics: Dextromoramide and fentanyl. Europ. J. Pharm. 1974, 28, 66-75.

16. Lusa F. T., Amaral R. V.: Remove from marked records bilateral entropion: a brief review. Pubvet. 2010, 4, 10.

17. Maggs D. J., Miller P. E., Ofri R.: Slatter's Fundamentals of Veterinary Ophthalmology. Elsevier Saunders, St. Louis (MO) 2013.

18. Nijakam I. N., Huault M., Prison V., Detilleux J.: The influence of phylogenic origin on the occurrence of Brachycephalic Airway Obstruction Syndrome in large retrospective study. Intern. J. Appl. Res. Vet. Med. 2009, 7, 138-143.

19. Nishiyama T., Yokoyama T., Hanaoka K.: Effects of sevoflurane and isoflurane anesthesia on arterial ketone body ratio and liver function. Acta. Anaesth. Scand. 1999, 43, 347-351.

20. Pichetto M., Arrighis S., Roccabianca P., Romussi S.: The Anatomy of the Dog Soft Palate. II. Histological Evaluation of the Caudal Soft Palate in Brachycephalic Breeds with Grade I Brachycephalic Airway Obstructive Syndrome. Anatom. Rec. 2011, 294, 1267-1272.

21. Read R., Broun H.: Entropion correction in dogs and cats using a combination Hotz-Celsus and lateral eyelid wedge resection: results in 311 eyes. Vet. Ophthalmol. 2007, 10, 6-11.

22. Riecks T. W., Birchard S. J., Stephens J. A.: Surgical correction of brachycephalic syndrome in dogs: 62 cases (1991-2004). J. Am. Vet. Med. Ass. 2007, 9, 1324-1328.

23. Ruffato M., Novello L., Clark L.: What is the definition of intraoperative hypotension in dogs? Results from a survey of diplomates of the ACVAA and ECVAA. Vet. Anaesth. Analg. 2014, 14, 1-10.

24. Topal A., Gül N., Ilçöl Y., Görgül O. S.: Hepatic effects of halothane, isoflurane or sevoflurane anaesthesia in dogs. J. Vet. Med. Physiol. 2003, 50, 530-533.

25. Torrez C. V., Hunt G. B.: Results of surgical correction of abnormalities associated with brachycephalic airway obstruction syndrome in dogs in Australia. J. Small Anim. Pract. 2006, 47, 150-154.

26. Wrzesińska A.: Przydatność wybranych parametrów klinicznych i badań dodatkowych oznaczanych u psów o morfotypie mesaticefalicznym i brachycefalicznym w okresie okołoznieczuleniowym przy operacjach okulistycznych. Praca doktorska. Biblioteka SGGW

Corresponding author: Agnieszka Wrzesińska, DVM, PhD, ul. Małcużyńskiego 7/4, 02-793 Warszawa; e-mail: agnieszka.wrzesinska17@gmail.com 